\title{
Provably secure group key management scheme based on proxy re-encryption with constant public bulletin size and key derivation time
}

\author{
GAURAV PAREEK*(D) and B R PURUSHOTHAMA \\ Department of Computer Science and Engineering, National Institute of Technology Goa, Farmagudi, \\ Ponda 403401, India \\ e-mail: gpareek@nitgoa.ac.in; puru@nitgoa.ac.in
}

MS received 23 May 2017; revised 1 January 2018; accepted 18 January 2018; published online 13 July 2018

\begin{abstract}
Users share a group key to decrypt encryptions for the group using a group key management scheme. In this paper, we propose a re-encryption-based group key management scheme, which uses a unidirectional proxy re-encryption scheme with special properties to enable group members share the updated group key with minimum storage and computation overhead. In particular, we propose a proxy re-encryption scheme that supports direct re-encryption key derivation using intermediate re-encryption keys. Unlike multihop re-encryption, the proposed proxy re-encryption scheme does not involve repeated re-encryption of the message. All the computations are done on the re-encryption key level and only one re-encryption is sufficient for making the group key available to the users. The proposed scheme is the first for group key management based on proxy re-encryption that is secure against collusion. The individual users store just one individual secret key with group key derivation requiring $\mathrm{O}(\log N)$ computation steps for a group of $N$ users. Size of the public bulletin maintained to facilitate access to the most recent group key for off-line members is $\mathrm{O}(N)$ and remains constant with respect to the number of group updates. The proposed group key management scheme confronts attacks by a non-member and even a collusion attack under standard cryptographic assumptions.
\end{abstract}

Keywords. Group key management; collusion-secure proxy re-encryption; logical key hierarchy; re-encryption key derivation; multi-cast security.

\section{Introduction}

Group communication, which finds application in services like teleconferencing, pay-TV, on-line games, etc. is for encrypting the messages sent to the group over a broadcast channel. Each group member decrypts the encrypted message using a group key (GK), which is shared securely with all the group members using a group key management (GKM) scheme. There may be changes to the group in an event of a member joining or leaving. The GK is updated so as to maintain backward and forward secrecy. A centralized group controller (GC) ensures that the updated GK is made available to all the group members. Consider a group $\mathcal{G}$ of $N$ members $\left\{U_{1}, U_{2}, \ldots, U_{N}\right\}$. In an event of a new user $u_{l}$ joining the group $\mathcal{G}, u_{l}$ must not be able to access messages sent to the group prior to its joining. This is called backward secrecy. For forward secrecy, a user $u_{l}^{\prime}$ who leaves the group $\mathcal{G}$, must not be able to decrypt any messages sent to $\mathcal{G}$ after it leaves. Maintaining forward and

*For correspondence backward secrecy while not subjecting the members and the GC to heavy computation burden and communication cost is a challenging task. In the remainder of the paper, we refer to the event of addition/removal of members to/from the group as group update. As a result of group update, the $\mathrm{GK}$ is updated by the GC, which is made available to group members using a message called re-key message. Using this re-key message, a member of the group can obtain the updated GK and auxiliary keys. A group member may miss a re-key message due to being off-line during a group update. Once the off-line member re-connects, she can obtain the required updated GK using a public bulletin maintained by the GC to store the re-key message(s) sent during the group update(s).

Performance of a GKM scheme is measured on the basis of storage and computation overhead on its members and GC due to various events in group communication. Storage is required for the secret and auxiliary keys of the members. Computation overhead is the number and nature of computations done by the group members to obtain the updated GK. Size of the public bulletin also constitutes storage cost and re-key message size sent by the GC constitutes computation overhead. 
There has been much research on these issues related with GKM. One of the solutions is logical key hierarchy (LKH) [1], in which each member of the group has to store $\log N^{1}$ secret auxiliary keys for a group of $N$ members. $\mathrm{LKH}$ requires all the re-key messages sent during the entire lifetime of the system, to be stored on the public bulletin. Group members willing to obtain updated GK from the public bulletin have to process all these re-key messages. This may lead to high storage and computation overhead for large groups if the group has a tendency to change frequently.

The afore-mentioned problem with the public bulletin size in LKH was addressed by Chen et al [2] using proxy re-encryption (PRE) [3, 4] for providing solution to GKM using LKH. Storage requirement for the public bulletin in the scheme due to Chen et al [2] is linear in the number of members in the group. It uses an RSA-based multi-hop unidirectional PRE scheme. A re-encryption key $r k_{i \rightarrow j}$ is assigned to each edge of the key tree between nodes labelled $i$ and $j$ such that node labelled $i$ is a parent of the node labelled $j$. GK encrypted for the root node of the hierarchy can be re-encrypted again and again until it is converted into a ciphertext under public key of the user associated with the leaf node. The scheme requires both RSA encryption and decryption keys to be stored secretly for each node. This increases storage overhead on the group members. However, the scheme was proved to be insecure against collusion attack by Chen and Tzeng [5], who also proposed a GKM scheme that is based on key management in hierarchy [6]. The scheme does not involve re-encryption and requires key derivation by a user. We notice that there is no GKM scheme that is provably secure against collusion attacks and is based on PRE. A collusion-resistant multi-hop re-encryption scheme $[7,8]$ requires repeated re-encryptions. Employing a unidirectional and multi-hop re-encryption scheme would require $\mathrm{O}(\log N)$ computationally costly reencryptions for a group of $N$ users.

\subsection{Our contributions}

To achieve constant number of re-encryptions in addition to constant sized re-key messages and linear public bulletin size, we propose a PRE scheme that supports derivation of a direct re-encryption key given a sequence of re-encryption keys. Using the proposed scheme, auxiliary re-encryption keys associated with edges in the key tree can be used to derive a direct re-encryption key from the root of the key tree to the leaf node associated with the group member. This direct re-encryption key can be used to reencrypt ciphertext containing the GK for the users. The corresponding user can decrypt the re-encrypted ciphertext

\footnotetext{
${ }^{1} \log _{d} N$ for a d-ary key tree. Key-trees of degree 2 are the most common.
}

to obtain the GK. The proposed scheme requires the users to store just one secret key. In the proposed scheme, each node of the key tree is assigned a key pair. Each directed edge is also assigned a re-encryption key, which is made public. Our contributions in the paper can be summarized as follows:

- We propose a re-encryption-based GKM scheme. The proposed PRE scheme for this purpose is used for key derivation in LKH. The GC performs just one encryption to encrypt the GK with the public key of the root node. Any group member can get the GK re-encrypted for itself using a direct re-encryption key from the root of the hierarchy to the node associated with the group member.

- This direct re-encryption key is derived by the group member using auxiliary publicly available re-encryption keys associated with the edges on the path from root to the leaf node associated with the group member.

- The proposed re-encryption scheme is a better option than the multi-hop re-encryption [7, 8] as it avoids repeated re-encryptions and all the computations are done on the re-encryption key level.

- In the proposed scheme, a re-key message sent as result of a group update has length $\mathrm{O}(\log N)$.

- The proposed GKM scheme is secure against collusion attacks and requires any off-line group member to execute $\mathrm{O}(\log N)$ steps to obtain the latest $\mathrm{GK}$ irrespective of the number of missed GK updates during its off-line time.

- Storage requirement for maintaining the public bulletin is linear with respect to the number of group members because only the most recent re-key message needs to be stored.

- Re-encryption keys associated with the edges can be made public and each user stores just its own secret key, which remains unchanged as long as she remains a member of the group.

- The GC does not have to store anything except the secret keys of the group members on its private storage.

\subsection{Organization of the paper}

Remainder of the paper is organized as follows. Section 2 presents a survey of the related research in the area of GKM. Section 3 presents a brief account of the LKH scheme. The proposed PRE scheme is defined in section 4 along with concrete description of its application for GKM. Security definitions and performance analysis are also given in section 4. Concrete construction of the proposed PRE scheme is presented in section $4.3 \mathrm{~b}$ along with detailed performance and security analysis. The paper finally concludes in section 5 . 


\section{Related work}

GKM has been extensively studied [1,9-16]. Among all these, the schemes based on LKH [1] are known for their efficiency in terms of the computation overhead on a group member for GK derivation. Users in LKH have to store $\log N$ auxiliary keys with the size of each re-key message being $2 \log N$. Several improvements have been proposed on the LKH scheme [17, 18]. One-way function tree (OFT) was the first improvement on the binary key tree, which reduced the number of re-key messages to $\log N$ by generating the parent key from the keys of the children [9]. Efficient Large-group Key (ELK) scheme [19], proposed with an aim to reduce re-key message size from $\log N$ to 0 , turns inefficient when a user joins the group, in which case, all the auxiliary keys are computed afresh. Mittra [15] divides the whole group into a collection of small subgroups. These subgroups have independent GKs. Therefore, joining or leaving of any group member affects just the subgroup key and its members while rest of all members remain unaffected. Such GKM schemes have been combined with PRE frameworks [11-13, 16, 20] so that instead of decrypting and then encrypting the data for the subgroup managers, they can directly re-encrypt the message without revealing the underlying keys. However, in all these schemes, the re-encryption keys are to be kept secret by the group managers. Chen et al [2] proposed a GKM based on PRE, which does not have this requirement. The groups are organized in a hierarchical structure as in LKH and the GK can be re-encrypted by anyone for the target user. However, the scheme was proved to be insecure against collusion attacks by Chen and Tzeng [5]. Also, to address challenge due to collusion attack, Chen and Tzeng [5] use key management in hierarchy [6] for managing access to the GK.

PRE [3] transforms ciphertext under a public key into a ciphertext under another public key. This transformation (re-encryption) can be carried out by a semi-trusted proxy using a special key called re-encryption key. Neither the reencryption key nor the ciphertexts reveal anything about the underlying plaintext. Also, secret keys of the delegator or delegatee are not disclosed even upon collusion between one of them and the proxy. Proxy cryptography was first introduced by Blaze et al [4] and further improved by Ivan and Dodis [21] to define important desirable properties. Ateniese et al [3] provide a list of desirable properties of PRE and proposed several PRE schemes, each satisfying different sets of properties. These properties are important to cryptographically delegate decryption rights for a piece of encrypted data in an application-specific manner. A collusion-free unidirectional multi-hop re-encryption scheme [7, 8] can be applied in LKH for GKM but has very high computation cost as it involves the costly re-encryption function to be computed several times. There is no PRE scheme in the literature that is efficient in terms of the number of re-encryptions required for re-encrypting a data item for a delegatee separated from the delegator by several hops.

\section{The LKH scheme}

The LKH scheme [1] is a widely studied GKM scheme using symmetric key cryptography. It uses a key structure as shown in figure 1a representing a key tree of degree 2 managing keys for $N=8$ group members $\left\{U_{1}, \ldots, U_{8}\right\}$. The GK is assigned to the key tree's root node. Auxiliary keys $\left\{K_{1}, \ldots, K_{14}\right\}$ are assigned to all the non-leaf nodes except the root node. These auxiliary keys help the group members for deriving GK. A group member has a unique leaf node corresponding to it. During a group update (as a result of member joining or leaving), auxiliary keys associated with the joining/leaving group member change to enable the group members obtain the updated GK (see figure $1 \mathrm{~b}$ and $1 \mathrm{c}$ ).

Let $\{M\}_{K}$ represent a symmetric encryption of $M$ using $K$ as the key. Each group member $U_{i}$ has $\mathrm{O}(\log N)$ auxiliary keys corresponding to nodes in the path from root of the key tree to the leaf node associated with $U_{i}$. For example, in figure 2, keys GK, $K_{14}, K_{12}$ and $K_{8}$ are associated with $U_{8}$. In an event of user $U_{8}$ leaving, GK and auxiliary keys $K_{14}$

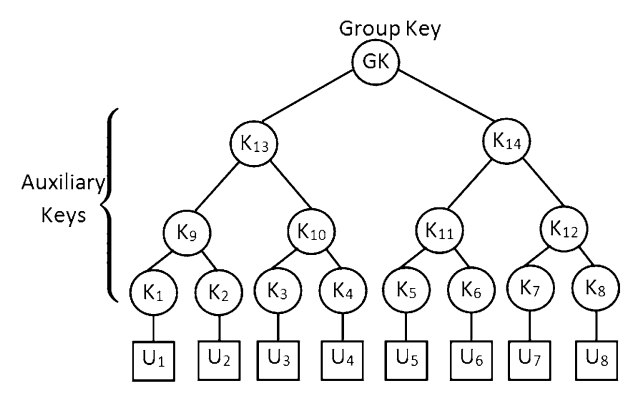

(a)

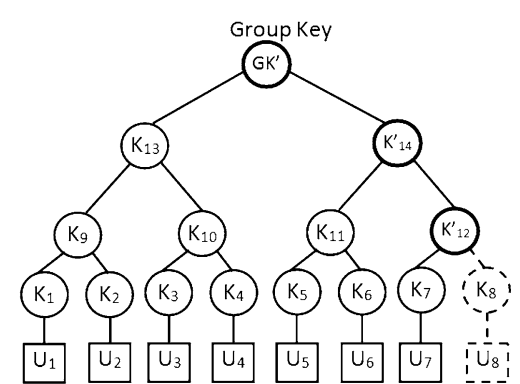

(b)

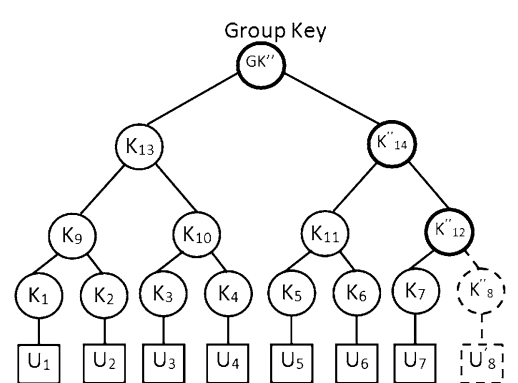

(c)

Figure 1. (a) Key assignment, (b) user leaving and (c) user joining in LKH. 


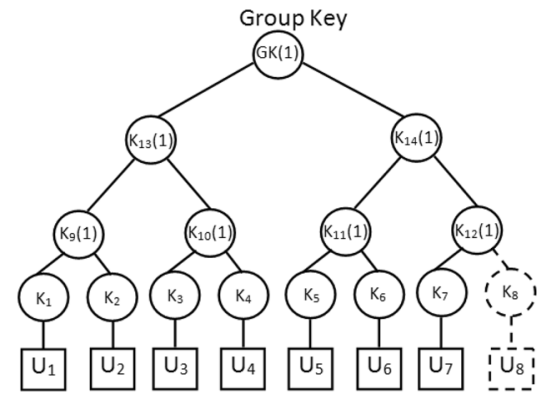

(a) Member $U_{8}$ goes off-line

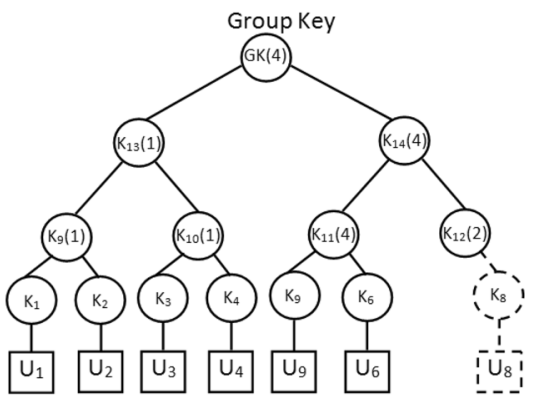

(d) Member $U_{9}$ joins the group

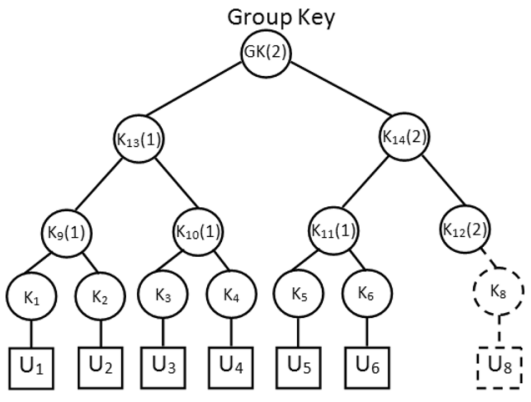

(b) Member $\mathrm{U}_{7}$ leaves the group

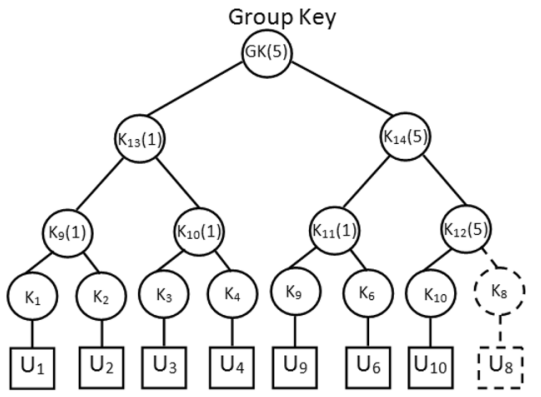

(e) Member $U_{10}$ joins the group

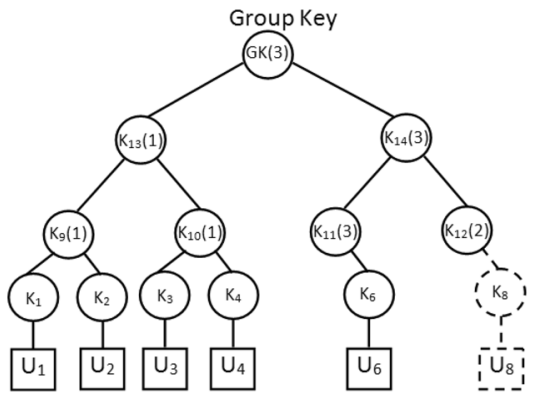

(c) Member $U_{5}$ leaves the group

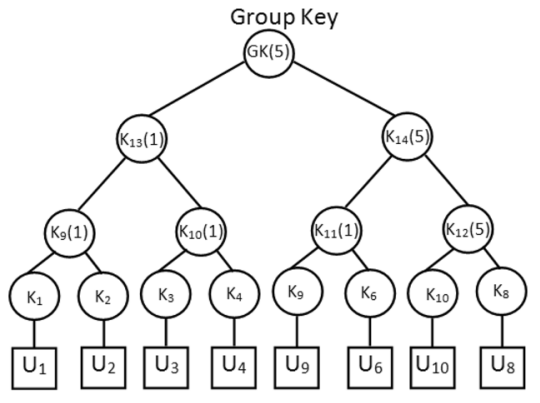

(f) Member $\mathrm{U}_{8}$ gets on-line again

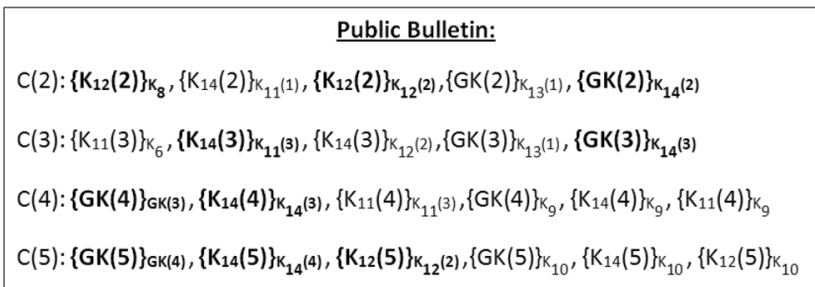

$\mathbf{K}_{\mathrm{i}}(\mathbf{j})$ is the key of associated with a node labeled $i$ at any time instant $j$

C(i) is the re-key message at any time instant $i$

Figure 2. Maintaining public bulletin in LKH for facilitating off-line members to derive group key.

and $K_{12}$ are updated. Since $K_{14}$ and $K_{12}$ are also associated with $U_{5}, U_{6}$ and $U_{7}$, the updated versions $K_{14}^{\prime}$ and $K_{12}^{\prime}$ are sent using $\left\{K_{12}^{\prime}\right\}_{K_{7}},\left\{K_{14}^{\prime}\right\}_{K_{11}}$ and $\left\{K_{14}^{\prime}\right\}_{K_{12}^{\prime}}$. Now that the users have their updated auxiliary keys, updated $\mathrm{GK}^{\prime}$ is sent using $\left\{\mathrm{GK}^{\prime}\right\}_{K_{13}}$ and $\left\{\mathrm{GK}^{\prime}\right\}_{K_{14}^{\prime}}$. The messages containing updated auxiliary keys and GKs are called re-key messages.

To handle the off-line group members who miss one or more GK updates, it maintains a public bulletin to store all the re-key messages. Upon re-connection, the group member processes all these re-key messages one at a time in the sequence they are stored and obtains the most recent auxiliary keys and the latest GK. Figure 2 shows one such scenario in which a group member $U_{8}$ goes off-line, after which two re-key messages $\mathrm{C}(2)$ and $\mathrm{C}(3)$ are sent as a result of $U_{7}$ and $U_{5}$ leaving the group. The group member $U_{8}$ also misses the re-key messages C(4) and C(5) sent when $U_{9}$ and $U_{10}$ join the group. All these re-key messages are stored on the public bulletin. When $U_{8}$ gets on-line, she needs the most recent GK. She obtains this by processing these entries on the public bulletin one at a time to obtain the most recent GK and the auxiliary keys using the steps as follows. First
$U_{8}$ processes the message $\mathrm{C}(2)$ to obtain the auxiliary keys $K_{12}(2), K_{14}(2)$ and $\mathrm{GK}(2)$. Using these updated auxiliary keys, $U_{8}$ processes $\mathrm{C}(3)$ to obtain $K_{14}(3)$ and $\mathrm{GK}(3)$. GK(4) and $K_{14}(4)$ are obtained from the message C(4). Finally, the most recent GK(5) and auxiliary keys $K_{12}(5)$ and $K_{14}(5)$ can be obtained by processing the message $C(5)$. Since the public bulletin must store all the re-key messages, public bulletin size must be on the order of the number of group updates. In other words, if $L_{\text {all }}$ is the number of users who left during the system's lifetime and $J_{\text {all }}$ the number of users who joined, the public bulletin size would be on the order of $\log N\left(L_{\text {all }}+J_{\text {all }}\right)$. This quantity grows with the number of changes in the group. Hence, if the group has a tendency to change very frequently, the public bulletin size is very high. Suppose $J_{\text {off }}$ is the number of group members who joined the group and $L_{\text {off }}$ is the number of users who left the group during off-line time of a user. The number of public bulletin messages processed by the off-line user after it gets on-line is on the order of $\log N\left(J_{\text {off }}+L_{\text {off }}\right)$.

In view of this discussion, it can be said that the LKH scheme does not suit groups that have high frequency of 
group updates. The proposed scheme features constant public bulletin size with respect to the number of group updates. Also, an off-line user has to do as many as $\log N$ computations when she gets on-line; this is again constant with respect to the number of group updates during a user's off-line period. The GC has to encrypt the GK just once and the number of computation steps required by individual group members to obtain the GK is $\mathrm{O}(\log n)$.

\section{Proposed PRE scheme}

The proposed GKM scheme uses unidirectional PRE scheme as a building block. The underlying PRE scheme must be unidirectional, key optimal, support public re-encryption keys and support derivation of a direct reencryption key given a sequence of intermediate re-encryption keys. The definition and construction of the concrete PRE scheme with its security analysis are presented. The proposed GKM scheme that uses the proposed PRE scheme is also presented with its security and performance analysis.

\subsection{Definition of the proposed PRE scheme}

A PRE scheme is used for transformation of a ciphertext intended directly for a user with public key $p k_{A}$ such that it becomes a ciphertext under $p k_{B}$. The proposed scheme differs from the existing ones in that in addition to re-encryption it also supports derivation of re-encryption key $r k_{A \rightarrow C}$ given $r k_{A \rightarrow B}, r k_{B \rightarrow C}$ and secret key $s k_{C}$.

Definition 1 We define the proposed PRE scheme as a collection $\Pi=$ (Setup, KeyGen, Enc, Dec ${ }_{1}$, ReKeyGen, ReEnc, Dec 2 , Derive) of algorithms defined as follows:

- Set-up $\left(1^{\lambda}\right) \rightarrow$ param: produces global system parameters param using security parameter $1^{\lambda}$ as input.

- KeyGen $(i) \rightarrow\left(s k_{i}, p k_{i}\right)$ : secret and public keys corresponding to the user $u_{i}$ are produced by this procedure.

- $\operatorname{Enc}\left(p k_{i}, m\right) \rightarrow C T_{i}$ : produces a ciphertext $C T_{i}$ under the public key $p k_{i}$ so that it can be decrypted using secret key $s k_{i} . C T_{i}$ can also be re-encrypted using appropriate re-encryption key(s).

- $\operatorname{Dec}_{1}\left(s k_{i}, C T_{i}\right) \rightarrow m$ : decrypts the ciphertext $C T_{i}$ using the secret key $s k_{i}$.

- ReKeyGen $\left(s k_{i}, s k_{j}\right) \rightarrow r k_{i \rightarrow j}$ : computes a re-encryption key $r k_{i \rightarrow j}$ to be used for transforming $C T_{i}$ into $C T_{j}$.

- $\operatorname{ReEnc}\left(r k_{i \rightarrow j}, C T_{i}\right) \rightarrow C T_{j}^{\prime}$ : this algorithm employs $r k_{i \rightarrow j}$ and the ciphertext $C T_{i}$ to obtain the re-encrypted ciphertext $C T_{j}^{\prime}$ under $p k_{j}$.

- $\operatorname{Dec}_{2}\left(s k_{i}, C T_{i}^{\prime}\right) \rightarrow m$ : decrypts the re-encrypted ciphertext $C T_{i}^{\prime}$ using secret key $s k_{i}$ of the intended user to obtain the underlying message $m$.
- $\operatorname{Derive}_{\mathbf{R K}}\left(s k_{n},\left\{r k_{1 \rightarrow 2}, \ldots, r k_{(n-2) \rightarrow(n-1)}, \rightarrow r k_{(n-1)} \rightarrow\right.\right.$ $n\}) \rightarrow r k_{1 \rightarrow n}$ : this procedure takes a sequence of one-hop re-encryption keys and secret key of the last user in the sequence as input to derive direct reencryption key from the first delegator to the last delegatee in the sequence.

In order for the re-encryption scheme defined earlier to be correct, following equalities must hold:

1. The procedure Dec $_{\mathbf{1}}$ is correct, that is, decryption of any direct ciphertext must give the correct underlying message.

$\operatorname{Dec}_{\mathbf{1}}\left(s k_{i}\right.$, Enc $\left.\left(p k_{i}, m\right)\right)=m$.

2. The procedure Dec $_{2}$ is correct, that is, decryption of a ciphertext re-encrypted using $r k_{i \rightarrow j}$ must give the correct underlying message upon decryption using $s k_{j}$ :

$\operatorname{Dec}_{2}\left(s k_{i}, \operatorname{ReEnc}\left(\operatorname{ReKeyGen}\left(s k_{i}, s k_{j}\right), \quad\right.\right.$ Enc $\left.\left.\left(p k_{i}, m\right)\right)\right)$ $=m$.

3. Derivation of direct re-encryption key given a sequence of one-hop intermediate re-encryption keys using the procedure Derive $\mathbf{R K}_{\mathbf{R K}}$ must be correct. That is

$\begin{array}{lll}\operatorname{Derive}_{\mathbf{R K}}\left(s k_{n}, \quad\left\{\operatorname{RekeyGen}\left(s k_{1}, s k_{2}\right),\right.\right. & \text { RekeyGen } \\ \left(s k_{2}, s k_{3}\right), \ldots, \quad \operatorname{RekeyGen}\left(s k_{(n-2)}, s k_{(n-1)}\right), & \text { RekeyGen } \\ \left.\left.\left(s k_{(n-1)}, s k_{n}\right)\right\}\right)=\operatorname{RekeyGen}\left(s k_{1}, s k_{n}\right) . & \end{array}$

\subsection{GKM scheme using the proposed PRE scheme}

In this section, we use the PRE scheme defined in the previous section to propose a GKM scheme. We present the key assignment and key derivation process for group members. The process carried out by the GC and the group members in case a member leaves or joins the group is also presented. Security and performance analysis of key derivation and in case of group update is also presented. We assume the existence of a publicly available cryptographic hash function $H: \mathcal{K} \rightarrow \mathcal{K}$ where $\mathcal{K}$ is the key space for the GKs. This hash function is used for updating the old GK to the newer version as part of the procedure for preserving backward secrecy. The desirable properties of $H$ include one-wayness, which provides pre-image resistance and collision resistance responsible for providing second preimage resistance.

4.2a Key assignment: Consider the key tree as shown in figure 3. Each node with label $v$ is assigned a key pair $\left(s k_{v}, p k_{v}\right)$ obtained using the procedure $\operatorname{KeyGen}(v)$ of $\Pi$. Each directed edge $(v, w)$ in the key tree is assigned a reencryption key $r k_{v \rightarrow w}$ such that node $v$ is the label of parent node of the node labelled $w$ in the key tree. Secret keys corresponding to all the nodes are stored by the GC. Key pair of each node of the key tree corresponds to the key pair of group members in the system. Figure 3 shows the key assignment when we apply PRE defined in section 4.1 to 


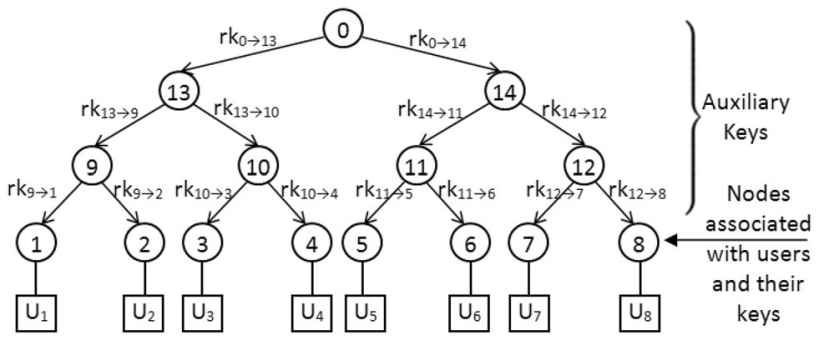

Figure 3. Assignment of key pairs (nodes) and auxiliary reencryption keys (edges) in the proposed scheme.

the GKM scenario in LKH. GC publishes all the auxiliary re-encryption keys assigned to edges.

4.2b Computing the GK: At any point in time, GC can use Enc procedure of $\Pi$ for encrypting the GK to obtain $C T_{0}=\operatorname{Enc}\left(p k_{0}, \mathrm{GK}\right)$ where $p k_{0}$ is the public key of the node labelled 0 . Now suppose user $U_{7}$ in figure 3 wants to access GK. The user initiates direct re-encryption key derivation process using its secret key $s k_{7}$ and re-encryption keys $r k_{0 \rightarrow 14}, r k_{14 \rightarrow 12}$ and $r k_{12 \rightarrow 7}$ corresponding to all the edges from the node labelled 0 down to the node labelled 7 . Thus, user $U_{7}$ obtains the re-encryption key $r k_{0 \rightarrow 7}$ using $\operatorname{Derive}_{\mathbf{R K}}\left(s k_{7},\left\{r k_{0 \rightarrow 14}, r k_{14 \rightarrow 12}, r k_{12 \rightarrow 7}\right\}\right)$. This $r k_{0 \rightarrow 7}$ is used to re-encrypt $C T_{0}$ into $C T_{7}^{\prime}$ using procedure $\operatorname{ReEnc}\left(r k_{0 \rightarrow 7}, C T_{0}\right)$. Now $C T_{7}^{\prime}$ can be decrypted by $U_{7}$ using procedure $\mathbf{D e c}_{2}\left(s k_{7}, C T_{7}^{\prime}\right)$ to obtain GK.

4.2c Member leaving: In an event of a member leaving the group, GK must be updated to $\mathrm{GK}^{\prime}$ (say) to preserve forward secrecy. The new GK has to be made available to all the users except the leaving user. For this, key pairs of all the nodes from the root down to the leaving node must be changed. For example, if the user $U_{8}$ leaves, key pairs of nodes labelled 0,14 and 12 must be updated to $\left(p k_{0^{\prime}}, s k_{0^{\prime}}\right),\left(p k_{14^{\prime}}, s k_{14^{\prime}}\right)$ and $\left(p k_{12^{\prime}}, s k_{12^{\prime}}\right)$, respectively, as shown in figure 4 . Then the GC computes new re-encryption keys for each edge involving the nodes whose key pair is updated. Hence, re-encryption keys $r k_{0^{\prime} \rightarrow 13}, r k_{0^{\prime} \rightarrow 14^{\prime}}$, $r k_{14^{\prime} \rightarrow 11}, r k_{14^{\prime} \rightarrow 12^{\prime}}$ and $r k_{12^{\prime} \rightarrow 7}$ are computed again using the updated key pairs of the nodes involved. GC now broadcasts $C T_{0^{\prime}}=\operatorname{Enc}\left(p k_{0^{\prime}}, \mathrm{GK}^{\prime}\right)$. The entry $C T_{0}$ is also replaced by $C T_{0^{\prime}}$ in the public bulletin. Now if any group member $\left(U_{1}\right.$ for example) wishes to access $\mathrm{GK}^{\prime}$, she can execute the procedure Derive $\mathbf{R K}_{\mathbf{R K}}\left(s k_{1},\left\{r k_{0^{\prime} \rightarrow 13}, r k_{13 \rightarrow 9}, r k_{9 \rightarrow 1}\right\}\right)$ to obtain a direct re-encryption key $r k_{0^{\prime} \rightarrow 1}$ from the root node to the node corresponding to $U_{1}$. This $r k_{0^{\prime} \rightarrow 1}$ can now be used for re-encrypting $C T_{0^{\prime}}$ into $C T_{1}^{\prime}$ using $\operatorname{ReEnc}\left(r k_{0^{\prime} \rightarrow 1}, C T_{0^{\prime}}\right)$. Group member $U_{1}$ can decrypt $C T_{1}^{\prime}$ using $\operatorname{Dec}_{2}\left(s k_{1}, C T_{1}^{\prime}\right)$ to obtain $\mathrm{GK}^{\prime}$.

Derivation of GK explained earlier is different from those of other PRE-based GKM schemes [2, 11, 20]. Unlike these schemes, the GK encrypted for the root node does not undergo repeated re-encryptions from root node to the leaf node associated with the user. In fact in the proposed scheme, re-encryption of the encrypted GK is carried out just once with other computations being done on the auxiliary keys associated with the edges in the key tree.

4.2d Member join: In case of a new member joining the group, the GC assigns key pair to the newly joining user. To maintain backward secrecy, nodes in the path from root node to the node corresponding to the joining user get "affected". Key pairs of all these affected nodes have to be updated. Suppose a user $U_{8^{\prime}}$ joins the group as shown in figure 5. The GC assigns a key pair $\left(s k_{8^{\prime}}, p k_{8^{\prime}}\right)$ to the newly joining user $U_{8^{\prime}}$, which is also the key pair for the node with label 8. Key pairs of the nodes labelled 0, 14 and 12 are also updated. As a result to the updated key pairs of the affected nodes, re-encryption keys $r k_{0^{\prime \prime} \rightarrow 14^{\prime \prime}}, r k_{14^{\prime \prime} \rightarrow 11}, r k_{14^{\prime \prime} \rightarrow 12^{\prime \prime}}, r k_{12^{\prime \prime} \rightarrow 7}$ and $r k_{12^{\prime \prime} \rightarrow 8^{\prime \prime}}$ are also updated using the procedure ReKeyGen. The GC now computes a new GK using the one-way hash function $H$ as $\mathrm{GK}^{\prime \prime}=H\left(\mathrm{GK}^{\prime}\right)$, notifies all the users with a single broadcast message about GK update and computes $C T_{0^{\prime \prime}}=\operatorname{Enc}\left(p k_{0^{\prime \prime}}\right.$, $\left.\mathrm{GK}^{\prime \prime}\right)$. All the on-line users update their copy of the GK using the same one-way hash function $H$ as $\mathrm{GK}^{\prime \prime}=H\left(\mathrm{GK}^{\prime}\right)$.

4.2e Updating auxiliary keys for off-line members: The GC maintains a public bulletin for storing updates missed by its members during their off-line time. Suppose a group member $U_{8}$ goes off-line for a period during which user $U_{7}$ leaves and user $U_{9}$ joins as shown in figure 6. As a result, $U_{8}$ misses the re-key messages sent by the GC as shown in figure $6 \mathrm{~b}$ and $\mathrm{c}$. The GC, however, stores the latest re-key message on the public bulletin as a result of latest group update. In figure 6 , when $U_{7}$ leaves the group, GK is updated to $\mathrm{GK}^{\prime}$ and the set of updated auxiliary re-encryption keys contains $r k_{0^{\prime} \rightarrow 13}, r k_{0^{\prime} \rightarrow 14^{\prime}}$, $r k_{14^{\prime} \rightarrow 11}, r k_{14^{\prime} \rightarrow 12^{\prime}}$ and $r k_{12^{\prime} \rightarrow 8}$. Another group update due to joining of the user $U_{9}$ involves updating the GK to GK". The set of updated auxiliary re-encryption keys contains $r k_{0^{\prime \prime} \rightarrow 13}, r k_{0^{\prime \prime} \rightarrow 14^{\prime \prime}}, r k_{14^{\prime \prime} \rightarrow 11}, r k_{14^{\prime \prime} \rightarrow 12^{\prime \prime}}$ and $r k_{12^{\prime \prime} \rightarrow 8}$. The GC now removes the previous contents stored on the public bulletin and stores the latest GK encrypted with public key of the root node $C T_{0^{\prime \prime}}=\operatorname{Enc}\left(p k_{0^{\prime \prime}}, \mathrm{GK}^{\prime \prime}\right)$ along with the updated public auxiliary re-encryption keys. When $U_{8}$ gets on-line again, she has to process contents of the public bulletin, which is nothing but the last re-key message for the group. The re-key message consists of the newest GK (GK') encrypted with the public key of the root node $C T_{0^{\prime \prime}}=\operatorname{Enc}\left(p k_{0^{\prime \prime}}, \mathrm{GK}^{\prime \prime}\right)$ and the last updated version of all the auxiliary keys modified in accordance with the procedure discussed in sections $4.2 \mathrm{c}$ and $4.2 \mathrm{~d}$. $U_{8}$ can obtain $\mathrm{GK}^{\prime \prime}$ using $C T_{0^{\prime \prime}}$ and the auxiliary re-encryption keys using the procedure described in section $4.2 \mathrm{~b}$.

To obtain the latest GK, a user who re-connects after missing a number of re-key messages has to process only $\log N$ auxiliary re-encryption keys. Hence, the amount of 


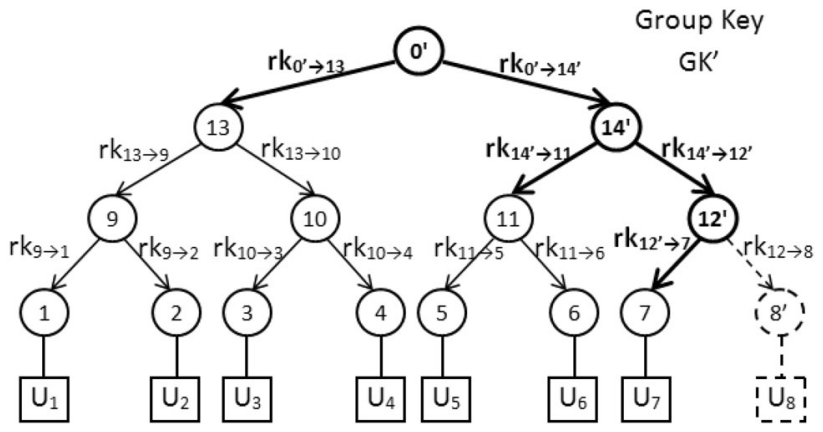

Figure 4. Re-key procedure when the user $U_{8}$ leaves the system.

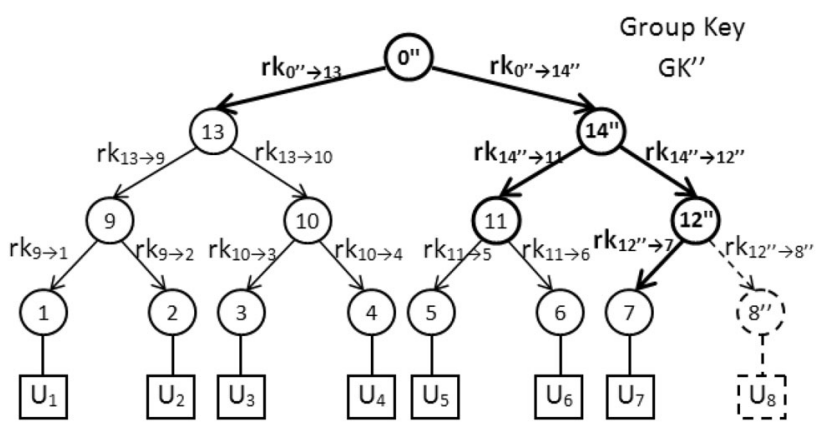

Figure 5. Re-key procedure when a user $U_{8}$ joins the system.

computation such a user has to do is independent of the number of join or leave events that take place during offline period of the user. Also, the size of re-key history to be maintained on the public bulletin by the GC is $\mathrm{O}(N)$, which is again independent of the number of group updates. In contrast, as explained in section 3, in LKH scheme [1], both public bulletin size and computation overhead on an off-line user increase with the number of group updates. As a result, if $J_{\text {all }}$ is the number of users that joined and $L_{\text {all }}$ is the number of users that left during a system's lifetime, size of the public bulletin in LKH is $\log N\left(J_{\text {all }}+L_{\text {all }}\right)$. Also, the number of re-key messages processed by the off-line user upon re-connection is $\log N\left(J_{\text {off }}+L_{\text {off }}\right)$. Here, $J_{\text {off }}$ and $L_{\text {off }}$ are the number of users that joined and left during the user's off-line time, respectively.

4.2f Performance analysis: The GC associates each member of the group with a leaf node of the key tree and all it has to store is her secret key. The re-encryption keys corresponding to the edges that fall on the path from root node to the leaf node corresponding to a group member constitute her auxiliary key components. These re-encryption keys can be made public, thereby not causing any storage overhead on the corresponding group members. Public keys of non-leaf nodes in the tree can also be stored on public storage space.
To access the GK, a group member has to compulsorily initiate re-encryption key derivation using the procedure Derive $_{\mathbf{R K}}$. The procedure Derive $\mathbf{R K}_{\mathbf{R}}$ takes reencryption keys corresponding to the edges that fall on the path from the root node down to the node associated with the user. The procedure Derive $\mathbf{R K}_{\mathbf{R K}}$ processes each reencryption key one at a time. Hence, the total number of operations is on the order of depth of the node corresponding to the group member. Now the re-encryption key obtained at the end of the Derive $\mathbf{R K}_{\mathbf{R K}}$ procedure can be used by anyone to re-encrypt the GK, which is further decrypted by the user to obtain GK. Since this reencryption key can also be made public, anyone can perform re-encryption. This relaxes the user of the burden of performing any computationally costly operations involved in re-encryption. Table 1 shows that each offline member who re-connects after some off-line period has to do only $\mathrm{O}(\log N)$ computation irrespective of the number of re-key updates missed by the off-line user. Also, the proposed scheme is secure against collusion attacks, which is proved in section 4.3e.

\subsection{Concrete proposed PRE scheme}

We have presented our GKM scheme based on a collusionresistant PRE, which supports re-encryption key derivation. This section presents the concrete construction of our proposed PRE scheme along with its security analysis.

4.3a Preliminaries: We define bilinear mapping, DLP and q-DBDHI assumptions.

Definition 2 Let $\mathbb{G}_{1}$ and $\mathbb{G}_{2}$ be two cyclic groups of prime order $p$. Let $g \in \mathbb{G}_{1}$ be the generator of $\mathbb{G}_{1}$; a bilinear map $[23,24] e: \mathbb{G}_{1} \times \mathbb{G}_{1} \rightarrow \mathbb{G}_{2}$ satisfies the following properties.

i. Bilinearity: $e\left(g^{l}, g^{k}\right)=e(g, g)^{l k}, \forall g \in \mathbb{G}_{1}, l, k \in \mathbb{Z}_{p}^{*}$.

ii. Non-degeneracy: $e(g, g) \neq 1$.

iii. Symmetric: $\quad e\left(g^{l}, g^{k}\right)=e\left(g^{k}, g^{l}\right)=e(g, g)^{l k}, \quad \forall$ $g \in \mathbb{G}_{1}, l, k \in \mathbb{Z}_{p}^{*}$.

iv. Computability: for computing the bilinear map, an efficient algorithm exists.

Definition 3 (Discrete Logarithm Problem (DLP) Assumption) Given the distributions $\left(g, g^{a}\right)$, the DLP assumption states that there does not exist a PPT algorithm that can efficiently compute the value of $a$.

Definition 4 (q-Decisional Bilinear Diffie-Hellman Inversion ( $q-D B D H I)$ Assumption) Given the distribution $\left(g, g^{m}, g^{n}, D\right)$, for $g \leftarrow \mathbb{G}_{1}, m, n \leftarrow \mathbb{Z}_{q}$ and $D \in \mathbb{G}_{2}$, the qDBDHI assumption states that it is computationally hard for a PPT algorithm to decide if $D=e(g, g)^{m / n}$. 


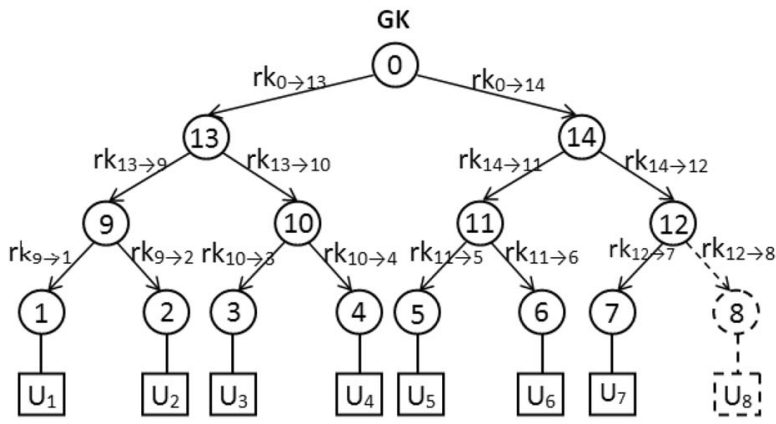

(a) Member $U_{8}$ goes off-line

Public bulletin:

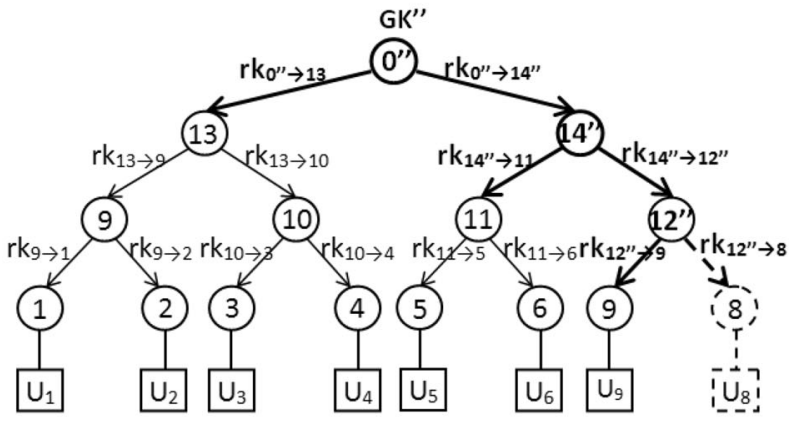

(c) Member $U_{9}$ joins the group

Public bulletin:

$\operatorname{Enc}\left(p k_{0^{\prime \prime}}, \mathrm{GK}^{\prime \prime}\right), \mathrm{rk}_{0^{\prime \prime} \rightarrow 13}, \mathrm{rk}_{0^{\prime \prime} \rightarrow 14^{\prime \prime}}, \mathrm{rk}_{14^{\prime \prime} \rightarrow 11}, \mathrm{rk}_{14^{\prime \prime} \rightarrow 12^{\prime \prime}}, \mathrm{rk}_{12} \rightarrow 8$

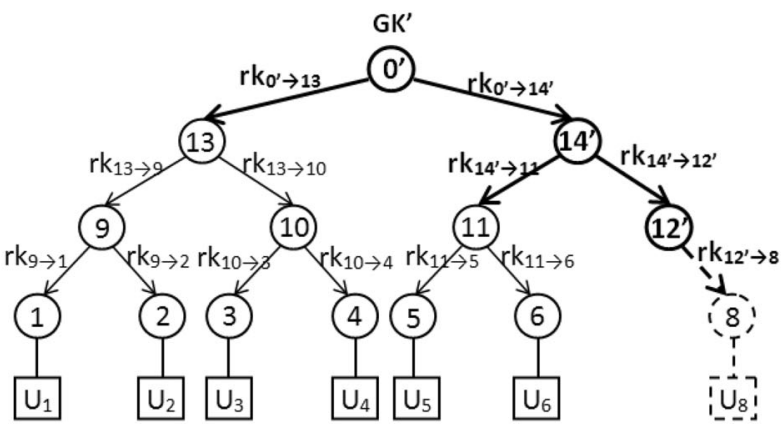

(b) Member $U_{7}$ leaves the group

\section{Public bulletin:}

$\operatorname{Enc}\left(p k_{0^{\prime}}, \mathrm{GK}^{\prime}\right), \mathrm{rk}_{0^{\prime} \rightarrow 13}, \mathrm{rk}_{0^{\prime} \rightarrow 14^{\prime}}, \mathrm{rk}_{14^{\prime} \rightarrow 11}, \mathrm{rk}_{14^{\prime} \rightarrow 12^{\prime}}, \mathrm{rk}_{12^{\prime} \rightarrow 8}$

Figure 6. Contents of the public bulletin in the proposed scheme (off-line case).

4.3b Concrete construction of proposed PRE scheme: We present construction of the proposed PRE scheme that supports direct re-encryption key derivation as defined in section 3. The scheme is semantically secure against a probabilistic polynomial time (PPT) adversary even under collusion. In this section, $a \in_{R} X$ the value $a$ being selected uniformly at random from the set denoted by $X$. The procedure Derive RK $_{\mathbf{R}}$ computes a direct multihop re-encryption key $r k_{1 \rightarrow n}$ given a sequence of $(n-1)$ one-hop re-encryption keys $\left\{r k_{1 \rightarrow 2}, \ldots, r k_{(n-1) \rightarrow n}\right\}$. In this section, description of Derive $\mathbf{R}_{\mathbf{R}}$ has been presented for a sequence of 2 one-hop re-encryption keys for 3 users. Extending the same description for a sequence of $(n-1)$ one-hop re-encryption keys for $n$ users is straightforward.

- Set-up $\left(1^{k}\right)$ : Taking security parameter $1^{k}$ as input, this algorithm determines public parameters: param = $\left(\mathbb{G}_{1}, \mathbb{G}_{2}, g, e, p\right)$ where $\mathbb{G}_{1}$ and $\mathbb{G}_{2}$ are distinct cyclic groups each of prime order $p, g \in \mathbb{G}_{1}$ is one of the

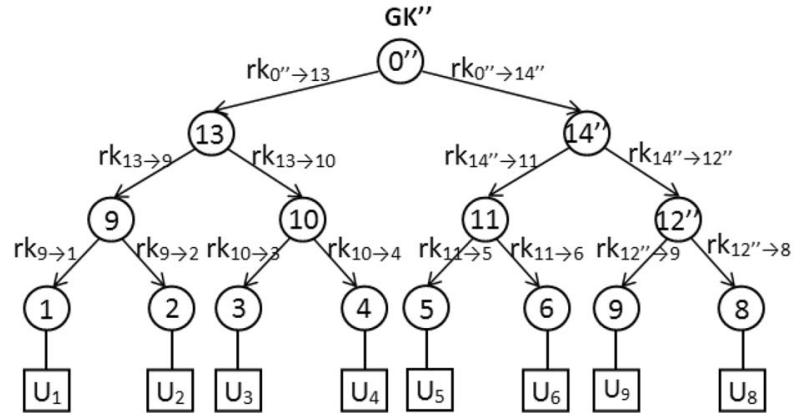

(d) Member $\mathrm{U}_{8}$ gets on-line again

Public bulletin:

$\operatorname{Enc}\left(p k_{0 "}, \mathrm{GK}^{\prime \prime}\right), \mathrm{rk}_{0 \prime} \rightarrow 13, \mathrm{rk}_{0 \prime} \rightarrow 14^{\prime \prime}, \mathrm{rk}_{14^{\prime \prime} \rightarrow 11}, \mathrm{rk}_{14^{\prime \prime} \rightarrow 12^{\prime \prime}}, \mathrm{rk}_{12 " \rightarrow 8}$ generators of $\mathbb{G}_{1}$ and a bilinear mapping $e: \mathbb{G}_{1} \times \mathbb{G}_{1} \rightarrow \mathbb{G}_{2}$.

- $\operatorname{KeyGen}(i$, param): This algorithm uses global parameters generated in the previous step to produce $\left(p k_{i}, s k_{i}\right)$ as follows:

- select $x_{i} \in_{R} \mathbb{Z}_{P}^{*}$ and set $s k_{i}=x_{i}$,

- select $y_{i} \in_{R} \mathbb{G}_{1}$,

- compute $\alpha_{i}=g^{x_{i}}, \beta_{i}=g^{1 / x_{i}} y_{i}$,

- set $p k_{i}=\left(\alpha_{i}, \beta_{i}\right)$,

- then key pair $\left(s k_{i}, p k_{i}\right)=\left(x_{i},\left(\alpha_{i}, \beta_{i}\right)\right)$.

- $\operatorname{Enc}\left(p k_{i}, m\right)$ : Any message $m \in \mathbb{G}_{2}$ can be encrypted under the public key $p k_{i}$ to produce $C T_{i}$ as follows:

- compute $A_{i}=e(g, g)^{t} m, B_{i}=\alpha_{i}^{t}$, where $t \in \in_{R} \mathbb{Z}_{p}$,

- then ciphertext $C T_{i}=\left(A_{i}, B_{i}\right)$.

- $\operatorname{Dec}_{1}\left(s k_{i}, C T_{i}\right)$ : This procedure obtains the underlying message $m$ from $C T_{i}$ using $s k_{i}$ :

- compute $A_{i} e\left(B_{i}, g^{1 / s k_{i}}\right)=m$. 


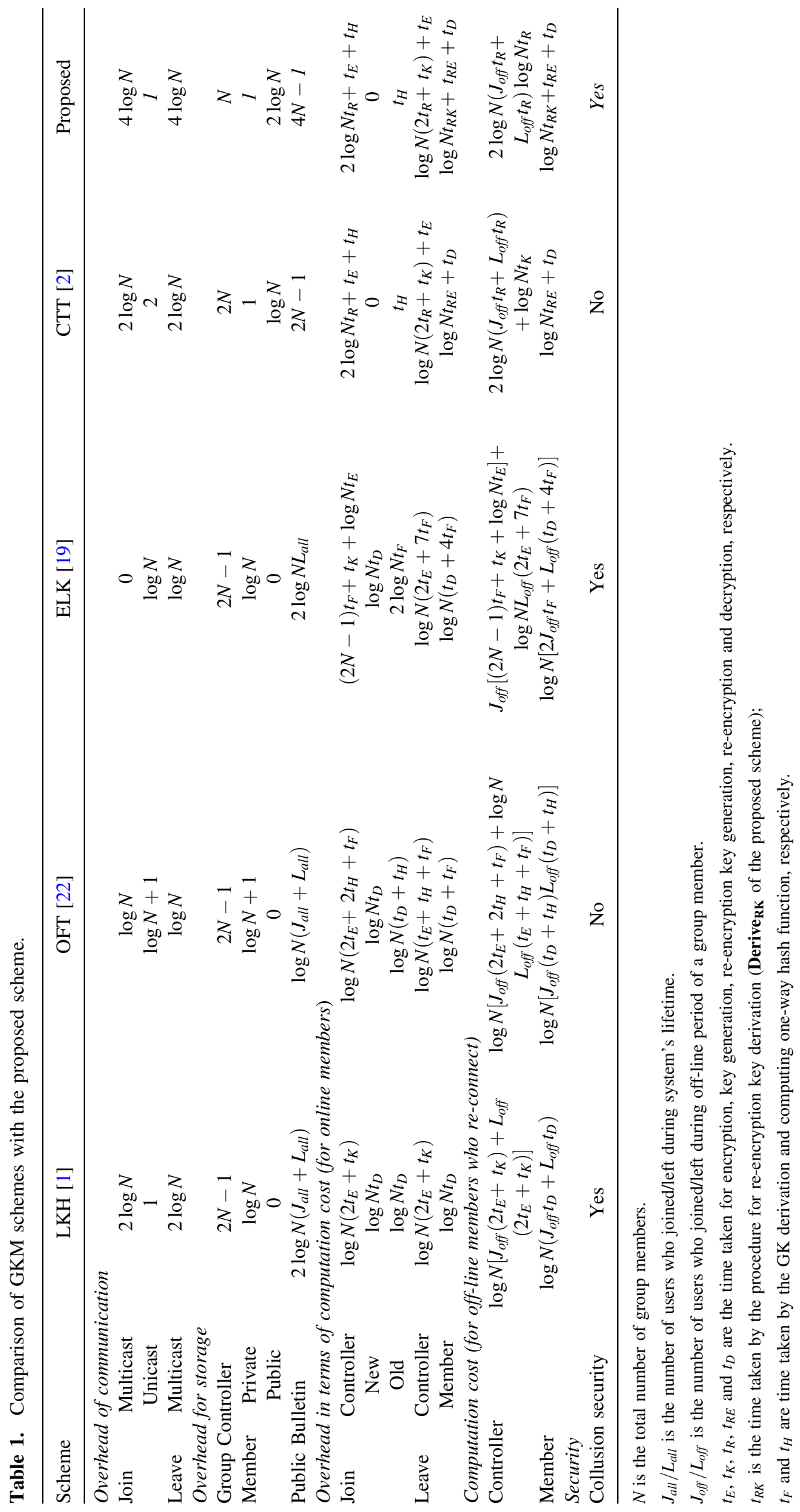


- ReKeyGen $\left(s k_{i}, s k_{j}\right)$ : A re-encryption key $r k_{i \rightarrow j}$ is generated using an interactive re-key generation algorithm as follows:

- compute $r k_{i \rightarrow j}^{(1)}=g^{x_{j} / x_{i}}, r k_{i \rightarrow j}^{(2)}=g^{y_{j} / x_{i}}$,

- set $r k_{i \rightarrow j}=\left(r k_{i \rightarrow j}^{(1)}, r k_{i \rightarrow j}^{(2)}\right)$.

- $\operatorname{ReEnc}\left(r k_{i \rightarrow j}, C T_{i}\right)$ : This procedure takes as input ciphertext containing the message encrypted under $p k_{i}$ and re-encryption key $r k_{i \rightarrow j}$ and computes reencrypted ciphertext under $p k_{j}$ using the steps as follows:

- compute $B_{j}^{\prime}=e\left(B_{i}, r k_{i \rightarrow j}^{(1)}\right)$,

- set $A_{j}^{\prime}=A_{i}$ and

- set $C T_{j}^{\prime}=\left(A_{j}^{\prime}, B_{j}^{\prime}\right)$.

- $\operatorname{Dec}_{2}\left(s k_{i}, C T_{i}^{\prime}\right)$ : This algorithm decrypts the re-encrypted ciphertext under public key $p k_{i}$ using $s k_{i}$ :

- compute $A_{i}^{\prime}\left(B_{i}^{\prime}\right)^{-s k_{i}}=m$.

- $\operatorname{Derive}_{\mathbf{R K}}\left(s k_{k},\left\{r k_{i \rightarrow j}, r k_{j \rightarrow k}\right\}\right)$ : This algorithm takes as input two one-step re-encryption keys and generates a two-step transitive re-encryption key. The algorithm works as follows:

- compute $y_{j}=\left(r k_{j \rightarrow k}^{(1)}\right)^{-1 / s k_{k}} \beta_{j}$,

- compute $r k_{i \rightarrow k}^{(1)}=\left(\left(r k_{i \rightarrow j}^{(2)}\right)^{y_{j}^{-1}}\right)^{s k_{k}}$.

The part of re-encryption key $r k_{i \rightarrow k}^{(1)}$ obtained here is sufficient for transformation of $C T_{i}$ into $C T_{k}^{\prime}$. Optionally, $r k_{i \rightarrow k}^{(2)}$ can also be computed as follows:

- compute $r k_{i \rightarrow k}^{(2)}=\left\{\left(r k_{i \rightarrow j}^{(2)}\right)^{1 / y_{j}}\right\}^{\beta_{k} g^{-1 / s k_{k}}}$.

- then $r k_{i \rightarrow k}=\left(r k_{i \rightarrow k}^{(1)}, r k_{i \rightarrow k}^{(2)}\right)$

4.3c Correctness of the concrete PRE scheme: The proposed PRE scheme satisfies the following correctness requirements:

1. Decryption of any directly encrypted ciphertext must correctly give the following message:

$\operatorname{Dec}_{1}\left(s k_{i}\right.$, Enc $\left.\left(p k_{i}, m\right)\right)=A_{i} e\left(B_{i}, g^{1 / s k_{i}}\right)$

$=m \cdot e(g, g)^{t} e\left(g^{x_{i} t}, g\right)^{-x_{i}^{-1}}=m$.

2. The procedure $\mathbf{D e c}_{2}$ is correct:

$\operatorname{Dec}_{2}\left(s k_{i}, \operatorname{ReEnc}\left(\operatorname{ReKeyGen}\left(s k_{i}, s k_{j}\right), \operatorname{Enc}\left(p k_{i}, m\right)\right)\right)$

$=\operatorname{Dec}_{2}\left(s k_{j},\left\{m e(g, g,)^{t}, e\left(g^{x_{j} / x_{i}}, g^{x_{i} t}\right)\right\}\right)$

$=m e(g, g)^{t}\left(e(g, g)^{x_{j} t}\right)^{-x_{j}^{-1}}=m$.

3. The procedure Derive $\mathbf{R K}_{\mathbf{R K}}$ is correct:

Derive $_{\text {RK }}\left(s k_{k},\left\{\right.\right.$ ReKeyGen $\left(s k_{i}, s k_{j}\right)$,

ReKeyGen $\left.\left.\left(s k_{j}, s k_{k}\right)\right\}\right)$

$=\left(r k_{j \rightarrow k}^{(1)}\right)^{-1 / s k_{k}} \beta_{j}=\left(g^{x_{k} / x_{j}}\right)^{-1 / x_{k}} g^{1 / x_{j}} y_{j}=y_{j}$.

Now, $y_{j}$ is used to compute:

$$
\begin{aligned}
& \left(\left(g^{y_{j} / x_{i}}\right)^{y_{j}^{-1}}\right)^{x_{k}}=g^{x_{k} / x_{i}}=r k_{i \rightarrow k}^{(1)} . \\
& \text { Also, }\left\{\left(r k_{i \rightarrow j}^{(2)}\right)^{1 / y_{j}}\right\}^{\beta_{k} g^{-1 / s k_{k}}}=\left\{\left(g^{y_{j} / x_{i}}\right)^{1 / y_{j}}\right\}^{g^{1 / x_{k}} y_{k} g^{-1 / x_{k}}} \\
& =g^{y_{k} / x_{i}}=r k_{i \rightarrow k}^{(2)} .
\end{aligned}
$$

4.3d Discussion: As we prove later in Theorem 1, the proposed re-encryption scheme is unidirectional and collusion-safe as it is computationally infeasible to obtain $r k_{j \rightarrow i}$ using the re-encryption key $r k_{i \rightarrow j}$ and all the public parameters. The scheme is interactive as it uses the secret keys of the communicating parties to generate re-encryption keys from one party to the other. Each user in the system has to store just one secret key irrespective of the number of delegations it accepts, meaning that the scheme is key-optimal. The scheme is non-transitive as one cannot obtain a direct re-encryption key $r k_{i \rightarrow k}$ given only the re-encryption keys $r k_{i \rightarrow j}$ and $r k_{j \rightarrow k}$. However, using $s k_{k}$, it is possible to obtain $r k_{i \rightarrow k}$ using the procedure Derive $\mathbf{R K}_{\mathbf{R K}}$. This $r k_{i \rightarrow k}$ avoids repeated re-encryptions of $C T_{i}$ into $C T_{j}$ and then into $C T_{k}$. In fact, $C T_{i}$ can be directly re-encrypted into $C T_{k}$ using ReEnc with $r k_{i \rightarrow k}$.

4.3e Security of the proposed GKM scheme: We prove security of the proposed PRE scheme against a PPT adversary $\mathcal{A}$ that tries to distinguish two direct or re-encrypted ciphertexts. We also prove that it is computationally infeasible for $\mathcal{A}$ to obtain the (master) secret key of any user given all the public keys, re-encryption keys and secret keys of the colluding users. We denote the set of key pairs of the honest users as $\left\{\left(s k_{B}, p k_{B}\right)\right\}$, set of key pairs of set of corrupted users as $\left\{\left(s k_{X}, p k_{X}\right)\right\}$ and key pair of the target user as $\left(s k_{i^{*}}, p k_{i^{*}}\right)$. Following is the definition for standard security of direct ciphertexts generated in the scheme:

$$
\begin{aligned}
& \operatorname{Pr}\left[\left(p k_{i^{*}}, s k_{i^{*}}\right) \leftarrow \operatorname{KeyGen}\left(i^{*}, \text { param }\right),\right. \\
& \left\{\left(p k_{B}, s k_{B}\right) \leftarrow \operatorname{KeyGen}(B, \text { param })\right\}, \\
& \left\{\left(p k_{X}, s k_{X}\right) \leftarrow \operatorname{KeyGen}(X, \text { param }),\right. \\
& \left\{r k_{B \rightarrow i^{*}} \leftarrow \operatorname{ReKeyGen}\left(s k_{B}, s k_{i^{*}}\right)\right\}, \\
& \left\{r k_{i^{*} \rightarrow B} \leftarrow \operatorname{ReKeyGen}\left(s k_{i^{*}}, s k_{B}\right)\right\}, \\
& \left\{r k_{X \rightarrow i^{*}} \leftarrow \operatorname{ReKeyGen}\left(s k_{X}, s k_{i^{*}}\right)\right\}, \\
& \left\{r k_{X \rightarrow B} \leftarrow \operatorname{ReKeyGen}\left(s k_{X}, s k_{B}\right)\right\}, \\
& \left(m_{0}, m_{1}, \alpha\right) \leftarrow \mathcal{A}\left(p k_{i^{*}},\left\{p k_{B}\right\},\left\{\left(p k_{X}, s k_{X}\right)\right\},\left\{r k_{B \rightarrow i^{*}}\right\},\right. \\
& \left\{r k_{i^{*} \rightarrow B}\right\},\left\{r k_{X \rightarrow i^{*}}\right\},\left\{r k_{X \rightarrow B}\right\}, C T_{i^{*}}, s \leftarrow\{0,1\}, \\
& \left.s^{\prime} \leftarrow \mathcal{A}_{k}\left(\alpha, \operatorname{Enc}\left(p k_{i}, m_{s}\right)\right): s=s^{\prime}\right]<\frac{1}{2}+\frac{1}{p o l y(k)} .
\end{aligned}
$$

This definition implies that a colluding adversary is able to distinguish two direct ciphertexts despite the availability of all the re-encryption keys in which delegator is honest, with negligible advantage. The following theorem proves security of the proposed PRE scheme according to this definition. 
Master secret security is defined as follows:

$$
\begin{aligned}
& \operatorname{Pr}\left[\left(p k_{i^{*}}, s k_{i^{*}}\right) \leftarrow \operatorname{KeyGen}\left(i^{*}, \text { param }\right),\right. \\
& \left\{\left(p k_{B}, s k_{B}\right) \leftarrow \operatorname{KeyGen}(B, \text { param })\right\} \\
& \left\{\left(p k_{X}, s k_{X}\right) \leftarrow \operatorname{KeyGen}(X, \text { param })\right\} \\
& \left\{r k_{B \rightarrow i^{*}} \leftarrow \operatorname{ReKeyGen}\left(s k_{B}, s k_{i^{*}}\right)\right\} \\
& \left\{r k_{i^{*} \rightarrow B} \leftarrow \operatorname{ReKeyGen}\left(s k_{i^{*}}, s k_{B}\right)\right\} \\
& \left\{r k_{X \rightarrow i^{*}} \leftarrow \operatorname{ReKeyGen}\left(s k_{X}, s k_{i^{*}}\right)\right\} \\
& \left\{r k_{X \rightarrow B} \leftarrow \operatorname{ReKeyGen}\left(s k_{X}, s k_{B}\right)\right\} \\
& \alpha \leftarrow \mathcal{A}\left(p k_{i^{*}},\left\{p k_{B}\right\},\left\{\left(p k_{X}, s k_{X}\right)\right\},\left\{r k_{B \rightarrow i^{*}}\right\}\right. \\
& \left.\left\{r k_{i^{*} \rightarrow B}\right\},\left\{r k_{X \rightarrow i^{*}}\right\}: \alpha=s k_{i^{*}}\right]<\frac{1}{p o l y(k)} .
\end{aligned}
$$

We prove security of the second level ciphertext and master secret key of a target user. This security proof is used for proving security of the GKM scheme against a PPT adversary.

Theorem 1 (Standard security) The scheme proposed in section $4.3 \mathrm{~b}$ is secure assuming the q-Decisional Bilinear Diffie-Hellman Inversion ( $q-D B D H I)$ [25] is hard in $\left(\mathbb{G}_{1}\right.$, $\left.\mathbb{G}_{2}\right)$, that is, given $\left(g, g^{u}, g^{v}, D\right)$, where $g \in \mathbb{G}_{1}, u, v \in \mathbb{Z}_{p}$ and $D \in \mathbb{G}_{2}$ decide if $D=e(g, g)^{u / v}$.

Proof Adversary $\mathcal{A}$ is given all the public keys in addition to secret keys of the colluding users and all the re-encryption keys with no dishonest delegatee. We assume the existence of a simulator $\mathcal{S}$ that can break the q-DBDHI assumption in $\left(\mathbb{G}_{2}, \mathbb{G}_{2}\right)$ for $\mathcal{A}$ and another simulator $\mathcal{R}$ that can generate re-encryption key from a delegator to a delegatee if the two public keys are given to it as input. It is also assumed that the simulator $\mathcal{R}$ does not reveal secret keys of either the delegator or delegatee. Encryption oracle $(\mathcal{E})$ is also available that can produce direct ciphertext to be the same as the one produced by the procedure Enc. Adversary queries the encryption oracle a finite number of times with a goal to distinguish two direct ciphertexts. Adversary $\mathcal{A}$ issues a finite number of requests that take the following form:

1. $\left\{r k_{A \rightarrow B} \leftarrow \mathcal{R}\left(p k_{A}, p k_{B}\right)\right\}$, collection of re-encryption keys from the corrupted users to the honest users.

2. $\left\{r k_{B \rightarrow i^{*}} \leftarrow \mathcal{R}\left(p k_{A}, p k_{i^{*}}\right)\right\}$, set of re-encryption keys from the honest users to the target user.

3. $\left\{r k_{i^{*} \rightarrow B} \leftarrow \mathcal{R}\left(p k_{i^{*}}, p k_{B}\right)\right\}$, set of re-encryption keys from the target user to the honest users.

4. $\left\{r k_{A \rightarrow i^{*}} \leftarrow \mathcal{R}\left(p k_{A}, p k_{i^{*}}\right)\right\}$, re-encryption keys from the corrupted users to the target user.

5. Direct ciphertexts $C T_{i} \leftarrow \mathcal{E}\left(p k_{i}, m_{j}\right) \forall i \in\{1,2, \ldots, l\}$, $\forall j \in\{1,2, \ldots, r\}$.

After the steps above are executed, $\mathcal{A}$ outputs a challenge of the form $\left(m_{0}, m_{1}, \tau\right)$ where $m_{0} \neq m_{1}$ are the two messages and $\tau$ is the internal state information. The challenger randomly selects $s \in\{0,1\}$ and computes the direct ciphertext as $C T_{i^{*}}^{(s)}=\left(c_{1}, c_{2}\right)=\left(Z^{u / v} m_{s}, g^{u}\right)$. The simulation is perfect as $u=x_{i^{*}} t$ and $v=x_{i^{*}}$ and the values $g^{v}=$ $g^{x_{i^{*}}}$ and $g^{u}=g^{x_{i^{*} t}}$ are publicly available and part of the direct ciphertext respectively. The challenger now sends $C T_{i^{*}}^{(s)}$ to $\mathcal{A}$ who uses the simulator $\mathcal{S}$ to guess $s^{\prime}$ and succeeds $\left(s=s^{\prime}\right.$ ) only if $\mathcal{S}$ distinguishes two q-DBDHI tuples with non-negligible advantage. But this is contradiction to the definition of q-DBDHI complexity assumption (see definition 4). Therefore, the PRE scheme proposed in section $4.3 \mathrm{~b}$ is secure against a PPT adversary who tries to distinguish two direct ciphertexts given q-DBDHI is hard in $\mathbb{G}_{1}, \mathbb{G}_{2}$.

Theorem 2 (Master-key security) (GK security) Consider key assignment to nodes and edges in the key tree of the proposed GKM scheme. Given a ciphertext $C T_{0}=\operatorname{Enc}\left(p k_{0}\right.$, $\mathrm{GK})$, public keys of all the nodes and all the re-encryption keys, an external PPT adversary $\mathcal{A}$ cannot obtain any of the node's secret key sk $k_{i}$ given the DLP assumption is hard in $\mathbb{G}_{1}$.

Proof Consider an adversary $\mathcal{A}$ who can recover the master secret key $s k_{i^{*}}=x_{i^{*}}$ of the target user $u_{i^{*}}$ with nonnegligible probability $\epsilon$, then we can build a simulator $S$ that can compute discrete logarithms with probability $\epsilon$. The adversary $\mathcal{A}$ interacts with the target user and obtains its public key $p k_{i^{*}}$ along with all the re-encryption keys involving the target user as either a delegator or delegatee, i.e., $r k_{i^{*} \rightarrow X}$ and $r k_{X \rightarrow i^{*}}$. We assume the existence of a simulator $\mathcal{R}$ that can generate re-encryption keys if public keys of delegator and the delegatee are provided as argument without revealing secret keys of either of them. That is, $r k_{i \rightarrow j} \leftarrow \mathcal{R}\left(p k_{i}, p k_{j}\right)$. Simulation of $\mathcal{S}$ works as follows:

1. If the public key $\left(g, g^{a}\right)$ in $\mathbb{G}_{1}$ is given as input, it outputs the target public key $p k_{i^{*}}=\left(g^{x_{i^{*}}}, g^{a}\right)$ where $s k_{i^{*}} \in R \mathbb{Z}_{p}^{*}$ and $a \in \mathbb{Z}_{p}$ is another secret information corresponding to $u_{i^{*}}$ with a condition that $a$ can be obtained if secret key $x_{i^{*}}$ is obtained.

2. Next, execute the following poly $(k)$ number of times:

(a) $\left\{r k_{i^{*} \rightarrow X} \leftarrow \mathcal{R}\left(p k_{i^{*}}, p k_{X}\right)\right\}$, collection of re-encryption keys with the target user as delegator and the corrupted users as delegatee. One such re-encryption key from user $u_{i^{*}}$ to the user $u_{X^{(j)}}$ is

$r k_{i^{*} \rightarrow X^{(j)}}=\left(g^{x} X^{(j)} / x_{i^{*}}, g^{y_{X^{(j)}} / x_{i^{*}}}\right)$.

(b) $\left\{r k_{X \rightarrow i^{*}} \leftarrow \mathcal{R}\left(p k_{X}, p k_{i}^{*}\right)\right\}$, collection of re-encryption keys from the corrupted users to the target user. One re-encryption key from one of the corrupted users $u_{X^{(j)}}$ to the user $u_{i^{*}}$ is $r k_{X^{(j)} \rightarrow i^{*}}=\left(g^{x^{*} / x_{X^{(j)}}}, g^{y_{i^{*}} / x_{X^{(j)}}}\right)$.

3. Eventually, the adversary $\mathcal{A}$ must output the guessed secret key $s k_{i^{*}}$ of the target user.

It can be seen that the simulation is perfect; therefore, $\mathcal{A}$ is able to obtain the master secret key $s k_{i^{*}}$ of the target user $u_{i^{*}}$ 
if $\mathcal{S}$ can solve DLP in $\mathbb{G}_{1}$. However, this contradicts the DLP assumption (see Definition 3).

Given these proofs for standard and master-secret security, we prove security of the proposed GKM scheme. Security against GK distinguishability under collusion, forward secrecy and backward secrecy is proved.

Theorem 3 The proposed GKM scheme based on PRE scheme of section $4.3 \mathrm{~b}$ is secure.

Proof We prove security against collusion attacks, groupkey security, forward and backward secrecy using our proof results in Theorems 1 and 2 .

Collusion-safety: Collusion-resistance of the proposed GKM scheme captures inability of an attacker who colludes with a subset of group members and tries to gain access to secret key of any of the non-compromised nodes in the key tree. Given the key assignment using the proposed PRE scheme, if a PPT adversary can obtain secret key of a node labelled $i$ in the key tree, she can obtain the secret key $s k_{i}$. However, in Theorem 2, the master secret security that captures the security of $s k_{i}$ of the underlying PRE scheme has been proved. Hence, by Theorem 2, we can conclude that no PPT adversary can obtain the secret key corresponding to the nodes in the key tree even if she colludes with one or more group members.

GK security: Any non-member should not be able to obtain the GK. Given the key assignment of the proposed GKM scheme using the PRE scheme given in section 4.2a, $C T_{0}$ associated with the root of the key tree of the group contains GK encrypted using Enc procedure of the construction given in section 4.3b. Hence, a GK can be obtained if the procedure Enc can be broken. However, in Theorem 1, the procedure Enc has been proved to be semantically secure against a PPT adversary. Hence, given the proof for standard security in Theorem 1, GK security of the proposed GKM scheme also stands proved.

Forward secrecy: Consider a group member $U_{8}$ leaving in figure 4, as a result of which the GK is updated to $\mathrm{GK}^{\prime}$. All the auxiliary re-encryption keys associated with $U_{8}$ and key pairs of the nodes that are in the path starting from the root node to the node labelled 8 also change. The new GK $\left(\mathrm{GK}^{\prime}\right)$ is encrypted using public key of the root of the key tree as $C T_{0}=$ Enc $\left(p k_{0}, \mathrm{GK}^{\prime}\right)$. The updated $\mathrm{GK}^{\prime}$ can be accessed by $U_{8}$ given that $U_{8}$ either decrypts $C T_{0}$ or reencrypts $C T_{0}$ such that it becomes a ciphertext under $p k_{8}$. However, $C T_{0}$ has been proved to be semantically secure in Theorem 1. Hence, $U_{8}$ cannot gain access to $\mathrm{GK}^{\prime}$ by decrypting $C T_{0}$ unless $s k_{0}$ is made available to $U_{8}$, which is impossible. Re-encryption of $C T_{0}$ such that it becomes a ciphertext under $p k_{8}$ can be done using $r k_{0 \rightarrow 8}$. Hence, $U_{8}$ can obtain $\mathrm{GK}^{\prime}$ through re-encryption if she can derive a correct $r k_{0 \rightarrow 8}$. However, as mentioned earlier, all the reencryption keys associated with $U_{8}$ are changed, thus forbidding the derivation of a correct $r k_{0 \rightarrow 8}$. Hence, the proposed GKM satisfies forward secrecy.
Backward secrecy: The requirements for backward secrecy defined in section 4.3 e must be satisfied in an event of member joining. Consider the example in figure 5, where $U_{8}$ joins the group. A cryptographic hash function $H$ is computed over the old GK to obtain the updated GK as $\mathrm{GK}^{\prime \prime}=H(\mathrm{GK})$. A new user can obtain the old GK only if she is able to compute the hash in the reverse direction. However, this is in contradiction with the assumption regarding the one-wayness of the cryptographic hash function $(H)$ assumed to be used for updating the GK. Hence, the proposed GKM scheme satisfies backward secrecy.

\subsection{Discussion}

Over the years, there have been many improvements in LKH scheme for GKM. Since the structure of the proposed scheme conforms with that of LKH, the proposed scheme can be adapted for most of the LKH-based schemes for GKM. The idea of using PRE for GKM has been extensively studied [11-13, 16, 20] but all of them require the PRE keys to be kept private. The first scheme that allows re-encryption keys to be published [2] used RSAbased multi-hop PRE scheme. The scheme was proved to be insecure under collusion attack by Chen and Tzeng [5]. However, we point out that in the scheme due to Chen and Tzeng [5], actual re-encryption does not take place and the scheme involves direct application of key management in hierarchy [6]. In contrast, our proposed scheme for GKM using PRE involves re-encryption just once and is secure against collusion. The only computational load on a user is that of the derivation of direct re-encryption key from root node to the leaf node associated with the user. A user does not have to perform re-encryption, which involves costly bilinear map operation. However, in the absence of a dedicated re-encrypting proxy, the users will have to perform just one bilinear pairing because number of re-encryptions in any case would be just one. Therefore, in the absence of a dedicated proxy server, efficiency of the proposed PRE scheme surpasses that of a unidirectional multi-hop PRE scheme [7, 8], in which as many as $\log N$ bilinear pairing operations would be performed for a group of $N$ members.

\section{Conclusion}

In this paper, we have proposed a GKM scheme for logical key hierarchies using PRE. In the proposed scheme, a group member processes $\log N$ public auxiliary keys to obtain the latest GK in a group of $N$ users. We have proved that the underlying PRE scheme is collusion resistant. The proposed scheme has a unidirectional procedure for deriving direct re-encryption key using a sequence of one-hop re-encryption keys. Earlier GKM schemes using PRE that is both 
unidirectional and multi-hop required repeated re-encryptions in order for a group member to obtain the GK. The proposed scheme avoids this as it requires only one reencryption after deriving a direct re-encryption key. The proposed scheme handles GK updates for the off-line users as well. For this, a public bulletin is maintained to store all the re-key messages. We have shown that the size of this public bulletin is constant with respect to the number of group updates and is linear in the number $(N)$ of group members. Also, any off-line group member has to perform constant $(\log N)$ number steps to obtain the latest GK when she re-connects.

\section{Acknowledgements}

This work is supported by the Ministry of Human Resource Development, Government of India.

\section{References}

[1] Wong C K, Gouda M and Lam S S 2000 Secure group communications using key graphs. IEEE/ACM Trans. Netw. 8(1): $16-30$

[2] Chen Y R, Tygar J D and Tzeng W G 2011 Secure group key management using uni-directional proxy re-encryption schemes. In: Proceedings of the 2011 IEEE International Conference on Computer Communications, INFOCOM'11, pp. 1952-1960

[3] Ateniese G, Fu K, Green M and Hohenberger S 2006 Improved proxy re-encryption schemes with applications to secure distributed storage. ACM Trans. Inf. Syst. Secur. 9(1): $1-30$

[4] Blaze M, Bleumer G and Strauss M 1998 Divertible protocols and atomic proxy cryptography. In: Advances in Cryptology-EUROCRYPT'98, pp. 127-144

[5] Chen Y R and Tzeng W G 2012 Efficient and provably-secure group key management scheme using key derivation. In: Proceedings of the 11th IEEE International Conference on Trust, Security and Privacy in Computing and Communications, pp. 295-302

[6] Atallah M J, Blanton M, Fazio N and Frikken K B 2009 ACM Transactions on Information and System Security. ACM Trans. Inf. Syst. Secur. 12(3): 18:1-18:43

[7] Shao J, Liu P, Cao Z and Wei G 2011 Multi-use unidirectional proxy re-encryption. In: Proceedings of the IEEE International Conference on Communications (ICC), pp. 1-5

[8] Wang H, Cao Z and Wang L 2010 Multi-use and unidirectional identity-based proxy re-encryption schemes. Inf. Sci. 180(20): 4042-4059

[9] Sherman A T and McGrew D A 2003 Key establishment in large dynamic groups using one-way function trees. IEEE Trans. Softw. Eng. 29(5): 444-458
[10] Canetti R, Garay J, Itkis G, Micciancio D, Naor M and Pinkas B 1999 Multicast security: a taxonomy and some efficient constructions. In: Proceedings of the IEEE International Conference on Computer Communications (INFOCOM'99), vol. 2, pp. 708-716

[11] Chiu Y P, Lei C L and Huang C Y 2005 Secure multicast using proxy encryption. In: Proceedings of the 7th International Conference on Information and Communications Security, ICICS 2005, pp. 280-290

[12] Han Y, Gui X, Wu X and Yang X 2011 Proxy encryption based secure multicast in wireless mesh networks. J. Netw. Comput. Appl. 34(2): 469-477

[13] Huang C Y, Chiu Y P, Chen K T and Lei C L 2007 Secure multicast in dynamic environments. Comput. Netw. 51(10): 2805-2817

[14] Hur J, Shin Y and Yoon H 2007 Decentralized group key management for dynamic networks using proxy cryptography. In: Proceedings of the 3rd ACM Workshop on QoS and Security for Wireless and Mobile Networks, pp. 123-129

[15] Mittra S 1997 Iolus: a framework for scalable secure multicasting. In: Proceedings of the Conference on Applications, Technologies, Architectures, and Protocols for Computer Communication, SIGCOMM' 97, pp. 277-288

[16] Mukherjee R and Atwood J W 2007 Scalable solutions for secure group communications. Comput. Netw. 51(12): 3525-3548

[17] Li X S, Yang Y R, Gouda M G and Lam S S 2001 Batch rekeying for secure group communications. In: Proceedings of the Tenth International Conference on World Wide Web, pp. 525-534

[18] Sun Y and Liu K J R 2007 Hierarchical group access control for secure multicast communications. IEEE/ACM Trans. Netw. 15(6): 1514-1526

[19] Penrig A, Song D and Tygar D 2001 Elk, a new protocol for efficient large-group key distribution. In: Proceedings of the 2001 IEEE Symposium on Security and Privacy, pp. 247-262

[20] Hur J, Shin Y and Yoon H 2007 Decentralized group key management for dynamic networks using proxy cryptography. In: Proceedings of the Third ACM Workshop on QoS and Security for Wireless and Mobile Networks, pp. 123-129

[21] Ivan A and Dodis Y 2003 Proxy cryptography revisited. In: Proceedings of the Network and Distributed System Security Symposium (NDSS)

[22] Ku W C and Chen S M 2003 An improved key management scheme for large dynamic groups using one-way function trees. In: Proceedings of the International Conference on Parallel Processing Workshops, pp. 391-396

[23] Dan B and Franklin M 2001 Identity-based encryption from the weil pairing. In: Advances in Cryptology-CRYPTO'O1, pp. 213-229

[24] Dan B and Franklin M 2003 Identity-based encryption from the weil pairing. SIAM J. Comput. 32(3): 586-615

[25] Dodis Y and Yampolskiy A 2005 A verifiable random function with short proofs and keys. In: Proceedings of the 8th International Workshop on Theory and Practice in Public Key Cryptography, pp. 416-431 